\title{
EXTRACCIÓN Y CARACTERIZACIÓN DEL ACEITE ESENCIAL DE Lippia origanoides H.B.K. "ORÉGANO DE MONTE" CULTIVADO EN EL QUINDÍO Y EVALUACIÓN DE LA ACTIVIDAD ANTIMICROBIANA
}

\section{EXTRACTION AND CHARACTERIZATION OF THE ESSENTIAL OIL OF Lippia origanoides H.B.K. "ORÉGANO DE MONTE" CULTIVATED AT QUINDÍO AND EVALUATION OF ANTIMICROBIAL ACTIVITY}

\author{
Julieth Henao ${ }^{1}$, Leidy J. Muñoz ${ }^{1}$, Leonardo Padilla ${ }^{2}$, Eunice Ríos ${ }^{1}$ \\ ${ }^{1}$ Laboratorio de Investigación Agroindustria de Frutas Tropicales, Programa de Química, Universidad del Quindío. \\ ${ }^{2}$ Grupo de Inmunología Molecuar (GYMOL). Universidad del Quindío.
}

Fecha de recibido: Febrero 3 de 2010

Fecha de aceptado: Junio 9 de 2010

Correspondencia: Maestría en Química, Universidad del Quindío. Av. Bolivar calle 12 norte Armenia Quindío. Correo electrónico:

erios@uniquindio.edu.co

\section{RESUMEN}

Se extrajo el aceite esencial de Lippia origanoides H.B.K. utilizando dos técnicas, Hidrodestilación Asistida por Microondas (MWHD) y Destilación por Arrastre con Vapor (DAV), con tiempos de operación óptimos de 60 y 90 minutos respectivamente, obteniendo mejores porcentajes de rendimiento para la MWHD, con hojas en estado seco. El análisis del aceite esencial por Cromatografía de Gases Acoplado a Detector de Masas mostró que el compuesto mayoritario fue el Timol. La actividad antimicrobiana del aceite esencial se evaluó en bacterias Gram-positivas y Gram-negativas, hongos filamentosos y levaduras, utilizando diferentes concentraciones de aceite, en porcentaje $V / V$ con etanol al 98\%. La acción antimicrobiana fue mayor en los microorganismos Gram-positivos yen los hongos y levaduras.

Palabras clave: aceite esencial, Hidrodestilación Asistida por Microondas, Destilación por Arrastre con Vapor, porcentaje de rendimiento, actividad antimicrobiana.

\section{ABSTRACT}

The essential oil from Lippia origanoides H.B.K. was extracted using two techniques, Microwave Assisted Hydrodistillation (MWHD) and Steam Distillation apparatus (SV), with optimal operation times of 60 and 90 minutes respectively, giving better yield rate for MWHD, with leaves in the dry state. The analyses of the essential oil by Gases Chromatography coupled with Mass Detector showed that majority compound was Thymol. The antimicrobial activity of essential oil was evaluated in Gram-positive and Gram-negative bacteria, filamentous fungus and yeast, using different concentrations of oil, in V/V percentage with $98 \%$ ethanol. The antimicrobial action was greater in the Gram-positive and fungus and yeast microorganism.

Key words: essential oil, Microwave Assisted Hydrodistillation, Steam Distillation, yeald rate, antimicrobial activity.

\section{INTRODUCCIÓN}

Los aceites esenciales son mezclas homogéneas de compuestos químicos orgánicos, provenientes de una misma familia química, terpenoides (1). Los aceites esenciales están contenidos en glándulas o vesículas secretoras inmersas en los tejidos de las hojas, flores, corteza y semillas de los frutos de muchas especies. Las plantas pueden producir aceite esencial para muchos y diversos fines; por un lado protegen a la planta de plagas, enfermedades e inclusive la invasión de otras plantas, para atraer insectos y aves (polinizantes) (2).
Los aceites esenciales de plantas aromáticas y medicinales presentan bioactividades notables (propiedades antifúngicas, antibacteriales y antioxidantes), atrayendo la atención de importantes sectores de la industria farmacéutica, de perfumes, cosmética y de alimentos, entre otras, por sus posibles y viables aplicaciones (3).

Lippia origanoides H.B.K., conocida como "Orégano de monte", es un arbusto silvestre, nativo de algunos países de América Central y del norte de América del Sur, especialmente en la Amazonía (4). Esta especie 
alcanza $3 \mathrm{~m}$ de longitud, posee hojas ovadas muy aromáticas e inflorescencias en racimo, axilares y blancas. La composición de los extractos de esta planta está sujeta a variables como: técnica de extracción, tratamiento o almacenamiento del material vegetal y condiciones geobotánicas de crecimiento (5). La infusión de sus hojas y flores es indicada para el tratamiento de dolor de estómago, indigestión, diarrea, acidez estomacal, nauseas, cólicos menstruales, y fiebre, así como antiséptico general para la boca, garganta y heridas, entre otros (4).

\section{MATERIALES Y MÉTODOS}

\section{Material vegetal}

Las hojas de L. origanoides H.B.K. fueron donadas por la Asociación Bioagroindustrial ASOBAI, ubicada en el corregimiento de Pueblo Tapao, en el municipio de Montenegro, departamento del Quindío. La planta se clasificó en el herbario de la Universidad del Quindío. La investigación se llevó a cabo en el Laboratorio de Investigación Diseño de Nuevos Productos de la Universidad del Quindío, Armenia, Colombia.

\section{Extracción del aceite esencial}

El aceite esencial de las hojas de $L$. origanoides, en estado fresco y seco, se obtuvo por dos métodos, Hidrodestilación Asistida por Microondas (MWHD) y Destilación por Arrastre con Vapor (DAV). Para la MWHD se empleó un horno microondas (Philips, 990 W) dentro del cual se colocó el balón con agua y material vegetal (100 g). La Destilación por DAV se llevó a cabo utilizando un equipo de destilación tipo Clevenger modificado, con $100 \mathrm{~g}$ de material vegetal. Ambos equipos se operaron por tiempos de 30, 60, 90 y 120 minutos. El aceite esencial se secó sobre sulfato de sodio anhidro y se almacenó en frascos viales ámbar a una temperatura de 4 으.

\section{Análisis fisicoquímicos y espectroscópicos}

Se determinó el rendimiento porcentual del aceite esencial obtenido de acuerdo al peso en gramos de las hojas y la cantidad de aceite extraído, comparando los valores obtenidos con respecto al método utilizado y al tiempo de operación. Se determinó la densidad a 25 으, el índice de refracción a 20 ㄷ C y el color de acuerdo al método sugerido por Food Chemical Codex (1996) (6), y el pH a 20ㄷ․ Se determinó la densidad por picnometría a 25 으.

Para la GC-MS se se diluyó una alícuota de $50 \mu \mathrm{L}$ de aceite en $1 \mathrm{~mL}$ de diclorometano y se utilizó un cromatógrafo de gases HR-GC Konik 4000B acoplado a un detector de masas Q-12, con una columna capilar apolar de sílice fundida, con fase estacionaria de $5 \%$

Rev. Invest. Univ. Quindío (21): 82- 86. Armenia - Colombia
Fenil-poli (metilsiloxano) de $60 \mathrm{~m} \times 0.25 \mathrm{~mm}$, D.I. $\times 0.25$ $\mu \mathrm{m}$, utilizando Helio de grado 5,0 UAP como gas de arrastre, con el siguiente gradiente de temperatura: $2 \mathrm{~min}$ a $150^{\circ} \mathrm{C}$, aumento $10^{\circ} \mathrm{C} / \mathrm{min}$ hasta $320^{\circ} \mathrm{C}$ donde se mantiene $5 \mathrm{~min}$, el detector en modo de impacto electrónico (EI) inició luego de $3 \mathrm{~min}$ de inyección escaneando cada $0.5 \mathrm{seg}$ masas entre los 70 y $500 \mathrm{~m} / \mathrm{z}$.

\section{Actividad antimicrobiana}

La actividad antibamicrobiana del aceite esencial se realizó siguiendo la técnica de difusión en disco de Kirby - Bauer modificado. Los microorganismos evaluados fueron: Escherichia coli, Pseudomonas aeruginosa, Aeromona hydrophila, Proteus mirabilis, Enterobacter cloacae, Klebsiella Pneumoniae (bacterias Gram-negativas, Staphylococcus aureus y Enterococcus gallinarum (bacterias Gram-positivas, Candida albicans (levadura) y Aspergillus niger (hongo filamentoso). El control positivo utilizado fue Amikacina $(30 \mu \mathrm{g}$ ) y el control negativo Etanol (98\%). Las placas se incubaron a 37 으 de 24 h a 7 días, dependiendo del microorganismo, y después de este tiempo se midió el diámetro de la zona de inhibición (mm).

\section{RESULTADOS Y DISCUSIÓN}

\section{Análisis fisicoquímicos y espectroscópicos}

La extracción del aceite esencial por los métodos de MWHD y DAV tuvo rendimientos óptimos para tiempos de 60 y 90 minutos de operación respectivamente (Fig. 1), sin embargo la primera técnica, además de utilizar menos tiempo de extracción, mostró un mayor porcentaje de rendimiento, lo cual implica una reducción en los costos de producción, esto por debido a que, como se ha reportado anteriormente (7), las microondas involucran un flujo de calor más eficiente, pues a diferencia de los métodos clásicos de calentamiento conductivo, las microondas pueden calentar toda la muestra casi simultáneamente y a un ritmo alto.

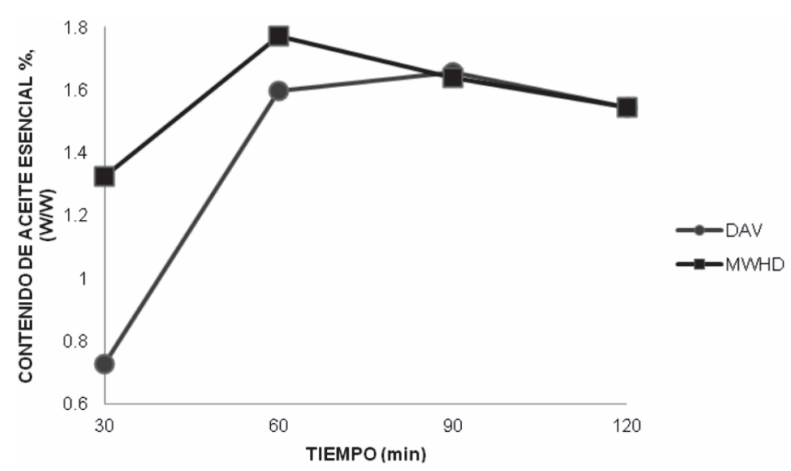

Figura 1. Comparación de las extracciones realizadas por DAV y por MWHD con respecto al tiempo de operación. 
Se hace una comparación entre los datos de las propiedades estándar con los datos obtenidos.

Las propiedades fisicoquímicas (apariencia, índice de refracción y peso específico) del aceite esencial de Lippia origanoides H.B.K. se muestran en la Tabla 1.
Para hacer una comparación, las propiedades estándar de las propiedades obtenidas de FFC (1996) se muestran también en la misma tabla. Los valores de estas propiedades se muestran dentro de los rangos especificados. El pH fue de 4.43.

Tabla 1. Propiedades fisicoquímicas del aceite esencial

\begin{tabular}{l|l|l}
\hline $\begin{array}{l}\text { Propiedad } \\
\text { fisicoquímica }\end{array}$ & FCC* $^{*}$ & Extraído \\
\hline Índice de refracción & De 1.5020 a 1.5080 & 1.5158 \\
Peso específico & De 0.935 a 0.960 & 0.932 \\
Color & $\begin{array}{l}\text { De rojo amarillento a } \\
\text { marrón rojizo oscuro }\end{array}$ & $\begin{array}{l}\text { De amarillo oscuro a a } \\
\text { rojo amallento }\end{array}$ \\
\hline
\end{tabular}

* Propiedades físicas estándar para el aceite esencial de orégano del FFC (1996).

El análisis cromatográfico mostró que el aceite esencial es rico en monoterpenos fenólicos, ya que el Timol fue el compuesto mayoritario, con una abundancia del
$29.6 \%$. Los componentes del aceite esencial de $L$. origanoides se encuentran en la Tabla 2.

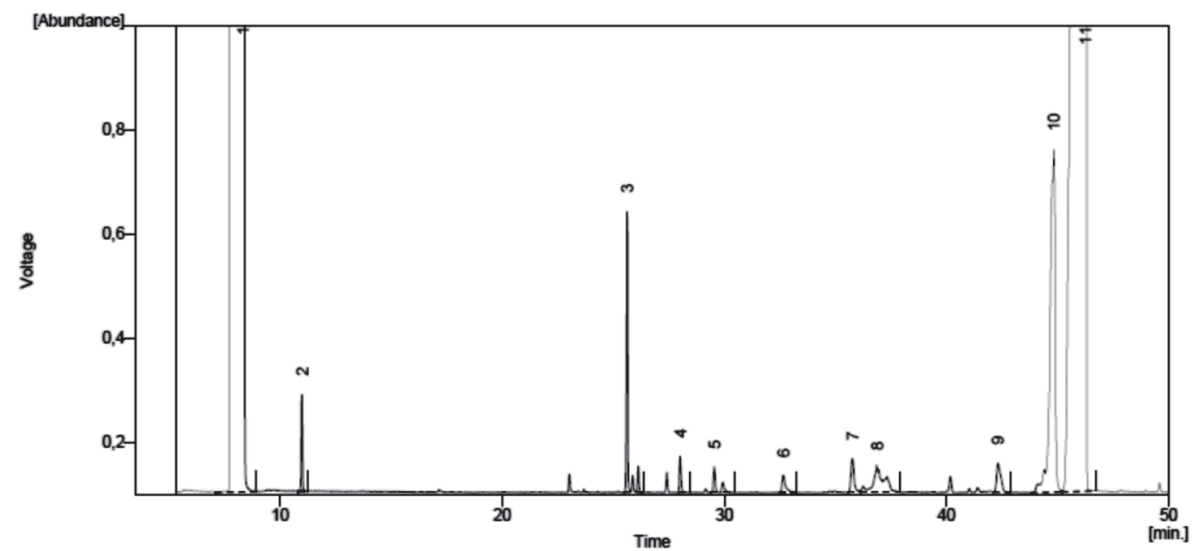

Figura 2. Cromatograma del aceite esencial obtenido por MWHD en un tiempo de 90 minutos.

Los compuestos del aceite esencial extraídos fueron identificados por la comparación de los modelos de fragmentación de sus espectros de masa con aquellos de compuestos similares de una base de datos. Para cada compuesto del cromatograma, el porcentaje de área de pico relativa a las áreas de los picos totales de todos los compuestos fue determinada y reportada como abundancia relativa de ese compuesto.

Tabla 2. Compuestos identificados en el aceite esencial de Lippia origanoides empleando CG-MS

\begin{tabular}{llcc}
\hline № & Compuesto & Tiempo de retención $(\mathrm{min})$ & Área - \% abundancia \\
\hline 1 & o-cimeno & 11.808 & 0.8 \\
2 & -pineno & 13.003 & 0.1 \\
3 & Terpineol & 13.775 & 0.1 \\
4 & 1-terpinen-4-ol & 15.328 & 0.1 \\
5 & Citroneleno & 16.882 & 0.2 \\
6 & Ácido-3-isopropilbenzóico & 17.439 & 0.6 \\
7 & Carvona & 20.160 & 0.2 \\
8 & Durenol & 21.417 & 3.5 \\
9 & Timol & 22.126 & 29.6 \\
10 & -ocimeno & 26.903 & 0.1 \\
\hline
\end{tabular}




\section{Actividad antimicrobiana}

Se encontró que la Klebsiella pneumoniae fue resistente frente a todas las concentraciones del aceite esencial. La Candida albicans y el Aspergillus niger tuvieron halos de inhibición de gran tamaño, y la inhibición de su crecimiento ocurrió a concentraciones hasta del $1 \%$ y el $10 \%$ respectivamente. De igual manera, la acción inhibitoria sobre las bacterias Gram-positivas fue mayor que la de las Gram-negativas, lo que se explica por la diferencia en la composición de la membrana de estos microorganismos. Esta fuerte acción antimicrobiana puede deberse a la acción de los terpenoides, ya que se ha reportado que estos compuestos son activos contra bacterias, hongos y protozoos, sin embargo su mecanismo de acción aun no esta completamente comprendido, aunque se cree que involucra el rompimiento de la membrana por los compuestos lipofílicos (8). Además, en otros estudios referentes al Timol se ha demostrado que este terpenoide tiene la capacidad de interactuar con las membranas fosfolipídicas, alterando la permeabilidad de la membrana del microorganismo (9).

Tabla 3. Zonas de inhibición del crecimiento de los microorganismos ( $\mathrm{mm}$ )

\begin{tabular}{|c|c|c|c|c|c|c|c|c|c|c|}
\hline \multirow[t]{2}{*}{ Microorganis mo } & \multicolumn{10}{|c|}{ Diámetro del halo de inhibición (mm) } \\
\hline & $1 \%$ & $5 \%$ & $10 \%$ & $20 \%$ & $30 \%$ & $40 \%$ & $50 \%$ & $70 \%$ & $\begin{array}{l}\text { Amikacin } \\
\text { a } 30 \mu \mathrm{g}\end{array}$ & $\begin{array}{l}\text { Etanol } \\
98 \%\end{array}$ \\
\hline $\begin{array}{l}\text { Enterococcus } \\
\text { gallinarum }\end{array}$ & $\mathrm{N}$ & $\mathrm{N}$ & $\mathrm{N}$ & 18 & 20 & 25 & 27 & 34 & 20 & $\mathrm{~N}$ \\
\hline $\begin{array}{l}\text { Staphyloccocus } \\
\text { aureus }\end{array}$ & $\mathrm{N}$ & $\mathrm{N}$ & 14 & 20 & 25 & 32 & 37 & 50 & ---- & $\mathrm{N}$ \\
\hline $\begin{array}{l}\text { Aeromona } \\
\text { hydropila }\end{array}$ & $\mathrm{N}$ & $\mathrm{N}$ & $\mathrm{N}$ & 10 & 15 & 23 & 28 & 33 & 17 & $\mathrm{~N}$ \\
\hline $\begin{array}{l}\text { Enterobacter } \\
\text { cloacae }\end{array}$ & $\mathrm{N}$ & $\mathrm{N}$ & $\mathrm{N}$ & 11 & 23 & 24 & 29 & 32 & 17 & $\mathrm{~N}$ \\
\hline Escherichia coli & $\mathrm{N}$ & $\mathrm{N}$ & $\mathrm{N}$ & 25 & 28 & 28 & 31 & 33 & 20 & $\mathrm{~N}$ \\
\hline $\begin{array}{l}\text { Klebsiella } \\
\text { pneumoniae }\end{array}$ & $\mathrm{N}$ & $\mathrm{N}$ & $\mathrm{N}$ & $\mathrm{N}$ & $\mathrm{N}$ & $\mathrm{N}$ & $\mathrm{N}$ & $\mathrm{N}$ & 19 & $\mathrm{~N}$ \\
\hline $\begin{array}{l}\text { Proteus } \\
\text { mirabillis }\end{array}$ & $\mathrm{N}$ & $\mathrm{N}$ & $\mathrm{N}$ & $\mathrm{N}$ & 17 & 18 & 26 & 31 & 20 & $\mathrm{~N}$ \\
\hline $\begin{array}{l}\text { Pseudomonas } \\
\text { aeruginosa }\end{array}$ & $\mathrm{N}$ & $\mathrm{N}$ & $\mathrm{N}$ & 10 & 24 & 26 & 26 & 30 & 22 & $\mathrm{~N}$ \\
\hline $\begin{array}{l}\text { Aspergillus } \\
\text { niger }\end{array}$ & $\mathrm{N}$ & $\mathrm{N}$ & 12 & 14 & 20 & 27 & 30 & 100 & ---- & $\mathrm{N}$ \\
\hline $\begin{array}{l}\text { Candida } \\
\text { albicans }\end{array}$ & 20 & 25 & 28 & 31 & 35 & 35 & 55 & 65 & ---- & $\mathrm{N}$ \\
\hline
\end{tabular}

\section{CONCLUSIONES}

El análisis de los métodos de extracción del aceite esencial demostró que la aplicación de la radiación microondas en el proceso aumenta los rendimientos; a partir del análisis cromatografico del aceite se estableció que el quimiotipo de la especie Lippia origanoides H.B.K. cultivada en el departamento del Quindío corresponde al del Timol, por ser su compuesto mayoritario, además de encontrar otros compuestos minoritarios del tipo terpeno fenólico. El aceite esencial de Lippia origanoides H.B.K. tiene un gran efecto de inhibición del crecimiento de los hongos y levaduras, seguido de las bacterias Grampositivas.

\section{AGRADECIMIENTOS}

Agradecemos a la Asociación Bioagroindustrial ASOBAl por el suministro del material vegetal, al Centro de Investigaciones Biomédicas de la Universidad del Quindío por la facilitación de los microorganismos, al Laboratorio de Análisis Instrumental de la Universidad del Quindío y al Laboratorio de Investigación Diseño de Nuevos Productos de la Universidad del Quindío. 


\section{BIBLIOGRÁFIA}

1. Cerpa, M. G. Hidrodestilación de Aceites Esenciales: Modelado y Caracterización. Tesis doctoral presentada para optar al grado de doctor de la Universidad de Valladolid.

2. Martínez, J., Sulbarán de Ferrer, B., Ojeda de Rodríguez, G., Ferrer, A. \& Nava R. Actividad Antibacteriana del Aceite Esencial de Mandarina. Revista de Facultad de Ciencias Agrónomas. 2003; (20):502-512.

3. Muñoz, A., Castañeda, M., Blanco, K., Cárdenas, C., Reyes, J.,Kouznetsob, V. \& Stashenko, E. Composición y Capacidad Antioxidante de Especies Aromáticas y Medicinales con Alto Contenido de Timol y Carvacrol. Scientia et Technica. 2007; (33): 125-128.

4. Oliveira, D.R., Leitão, G.G., Bizzo, H.R., Lopes, D., Alviano, D.S., Alviano, C.S. \& Leitão, S.G. Chemical and Antimicrobial Analyses of Essential Oil of Lippia origanoides H.B.K. Journal of food chemistry. 2007; (101): 236240.

5. Ruiz, C., Tunarosa, F., Martínez, J. \& Stashenko, E. Estudio Comparativo por GC-MS de Metabolitos Secundarios Volátiles de dos Quimiotipos de Lippia origanoides H.B.K., Obtenidos por Diferentes Técnicas de Extracción. Scientia et Technica. 2007; (33): 325-328.

6. FCC (1996). Food Chemical Codex (4th Ed.), pp. 482-483). Washington, DC: National Academic Press.

7. Golmakani, M-T., Rezaei, K. Comparison of Microwave-Assisted Hydrodistillation with the Traditional Hydrodistillation Method in the Extraction of Essential Oils from Thymus vulgaris L. Journal of Food Chemistry. 2007; 12 (84): 1-24.

8. Maguna, F.P., Romero, A.M., Garro, O.A. \& Okulik, N.B. Actividad Antimicrobiana de un Grupo de Terpenoides. Comunicaciones Científicas y Tecnológicas. 2006; E(57).

9. Trombetta, D., Castelli, F., Sarprieto, M.G., Venuti, V., Cristani, M., Daniele, C., Saija, A., Mazzanti, G. \& Bisignano, G. Mechanism of Antibacterial Action of Three Monoterpens. Journal of Antimicrobial Agents and Chemotheraphy. 2005; 49(6): 2474-2478. 